\title{
Acute Superior Mesenteric Venous Thrombosis in a Young Patient Without Risk Factors
}

Meghan E. Smith, MD; Katherine Percy, DO; Reis Brandon Ritz, MD

\section{In this case report, the authors address the diagnostic challenges of a young, healthy patient who presented to the ED with unrelenting abdominal pain.}

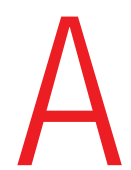

cute mesenteric ischemia (AMI) results when oxygen delivery to the mesenteric artery is compromised, and is a serious diagnosis that should be considered in patients of all ages to avoid significant morbidity and mortality. The majority of cases are due to arterial embolism, arterial thrombus, or intestinal hypoperfusion (non-occlusive). Acute mesenteric venous thrombosis (MVT) accounts for only $2 \%$ to $10 \%$ of AMI cases, and only $0.01 \%$ of emergency surgery admissions. ${ }^{1} \mathrm{~A}$ large systematic review showed a $44 \%$ mortality rate for MVT, in contrast to $66 \%$ to $89 \%$ for all other forms of AMI. ${ }^{2}$ The typical age range for MVT is reported between 45 and 60 years, with a slight male predominance. ${ }^{3}$ Dull, central abdominal pain is the most frequently reported symptom of MVT, although it is generally less impressive than the pain described in other forms of AMI. ${ }^{3}$ Along with the hallmark of abdominal pain out of proportion to the examination, other gastrointestinal symptoms include weight loss and non-specific altered bowel function (constipation, diarrhea, abdominal distention, and bloating), which are present in half of all patients with MVT. ${ }^{1}$ Peritoneal signs and bloody stools portend poor outcomes, as they often occur with disease progression. ${ }^{4}$

\section{Case}

A 26-year-old man presented to the ED with periumbilical and lower abdominal pain for 1 week. The pain was described as constant and dull, worsened by movement and oral intake, and improved with lying flat. He described bloating and decreased volume of bowel movements. He denied nausea, vomiting, fever, colicky pain, blood in stool, testicular pain, urinary complaints, trauma, or any similar episodes in the past. The patient had no known medical conditions or surgical history, except for a remote history of alcohol dependence (in remission) and tobacco use. There was no personal or family history of coagulopathy. Of note, he was seen by his primary care physician a few days

Dr Smith is a physician, department of emergency medicine and graduate medical education, Carl R. Darnall Army Medical Center, Fort Hood, Texas. Dr Percy is a physician, department of emergency medicine, Tripler Army Medical Center, Honolulu, Hawaii. Dr Ritz is a physician, department of emergency medicine, William Beaumont Army Medical Center, El Paso, Texas.

Authors' Disclosure Statement: The authors report no actual or potential conflict of interest in relation to this article. The views expressed are solely those of the authors and do not reflect the official policy or position of the US Army, the Department of Defense, or the US Government. 
prior to his ED presentation and had been instructed to take acetaminophen, which did not provide relief.

The patient's vital signs at presentation were: blood pressure, 122/70 $\mathrm{mm} \mathrm{Hg}$; heart rate, 93 beats/min; respiratory rate, 18 breaths $/ \mathrm{min}$; and temperature, $37.5^{\circ} \mathrm{C}\left(99.5^{\circ} \mathrm{F}\right)$. Oxygen saturation was $99 \%$ on room air. The physical examination was remarkable only for mild abdominal tenderness diffusely, greater in the lower and central abdomen than in the upper abdomen. The remainder of the physical examination was unremarkable.

Laboratory studies ordered included a complete blood count, comprehensive metabolic profile, lipase, and urinalysis. The patient did have a mild transaminitis (aspartate aminotransferase, $48 \mathrm{U} / \mathrm{L}$; alanine aminotransferase, $84 \mathrm{U} / \mathrm{L}$ ); the remainder of the studies were normal. A serum lactate, drawn after the $1 \mathrm{~L}$ of normal saline was administered intravenously (IV), was within normal limits $(0.7 \mathrm{mmol} / \mathrm{L})$. No prior laboratory studies were available for comparison.

The patient's continued abdominal pain and transaminitis prompted an ED bedside right upper quadrant ultrasound, which showed a small gallbladder polyp; no signs of gallbladder disease were present. The patient required three doses of morphine $4 \mathrm{mg}$ IV without complete pain relief. Given the concern for pain out of proportion to physical examination, a computed tomography (CT) scan of the abdomen/pelvis with IV and oral contrast was ordered. The radiologist interpreted the scan as showing a superior mesenteric vein (SMV) thrombus extending into the splenic/portal vein confluence and the intrahepatic portal veins (Figures 1 and 2). Mild mesenteric fat stranding secondary to edema was also present. Although there was no evidence of infarction or hemorrhage, the high risk of disease progression contributed to the decision to admit the patient. The patient was given a dose of enoxaparin and admitted to the hospital under the care of the medicine team.

Ciprofloxacin and metronidazole were administered IV for antibiotic prophylaxis, and the patient was placed on bowel rest with advancement to regular diet as tolerated. Propranolol was given for variceal prophylaxis. The patient was discharged home the following day in stable condition. Although he still had mild abdominal tenderness, the vital signs and physical examination were within normal limits. The patient was placed

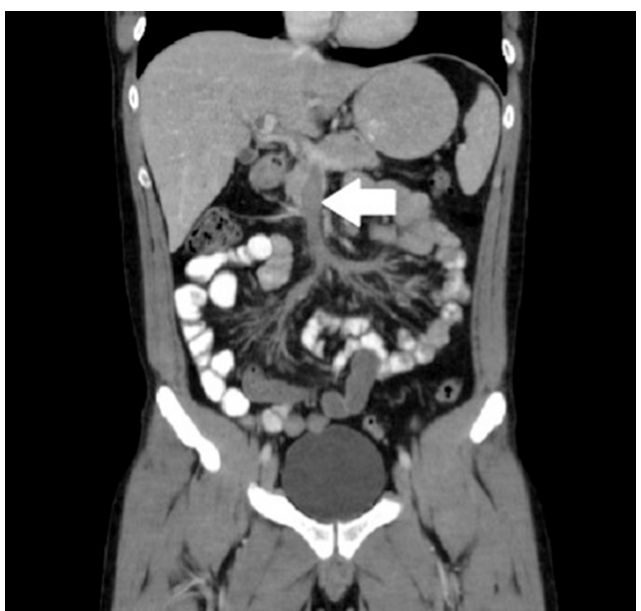

Figure 1. The arrow indicates a central low attenuation within a sharply defined enhanced venous wall, defining the thrombus in the superior mesenteric vein shown on computed tomography scan images.

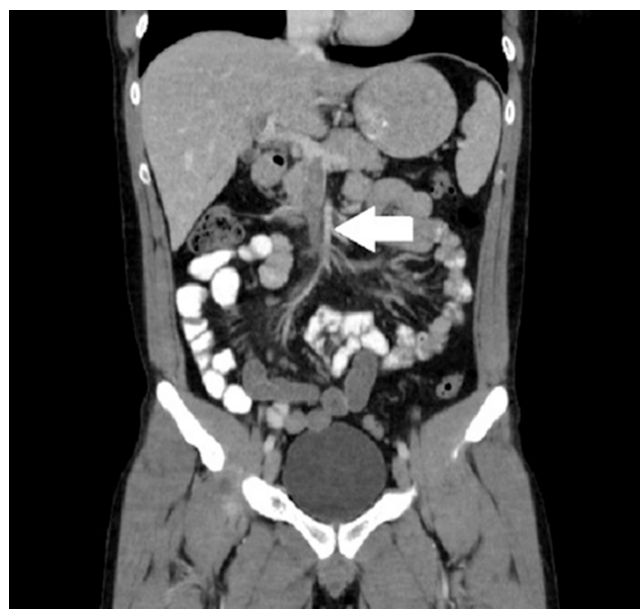

Figure 2. The arrow indicates superior mesenteric artery enhancement overlapping superior mesenteric venous thrombosis shown on computed tomography scan images. 
on a 6-month course of rivaroxaban therapy. Coagulopathy testing was scheduled at a later date, since ongoing anticoagulation treatment could interfere with test results. Unfortunately, the patient did not attend follow-up appointments to obtain testing.

\section{Discussion}

Mesenteric venous thrombosis is seen predominantly in middle-aged patients presenting with vague symptoms, which makes this a challenging diagnosis to make in the acute care setting. Risk factors for MVT include recent injury (causing trauma to the vasculature), recent surgery (causing stagnant blood flow), inflammatory conditions, and hypercoagulable states. ${ }^{1}$ In this patient's case, no risk factors were identified; although the majority of cases of MVT will have an identifiable risk factor. ${ }^{2}$ Still, $21 \%$ to $49 \%$ of cases of MVT are considered idiopathic. ${ }^{1,3}$ It is possible that our patient had a prior undiagnosed pancreatitis associated with his history of alcoholism that contributed to his thrombosis. Pancreatitis and other inflammatory conditions, including diverticulitis or inflammatory bowel disease, are more commonly associated with thrombus formation in the large veins, as opposed to an undiagnosed hypercoagulable state, which would more likely affect distal venuoles, vasa recta, or venous arcades. ${ }^{1,5}$ The patient's mild transaminitis was likely secondary to hepatic congestion from the venous thrombus extending to the splenic-portal vein confluence and intrahepatic portal vein. One study looked at patients with pancreatitis and found that $16.7 \%$ of their study population had an SMV thrombus, while $4.1 \%$ had a SMV thrombus with a concomitant portal vein thrombus. ${ }^{6}$

Although there are no pathognomonic laboratory findings of MVT, elevated lactate, leukocytosis, and elevated D-dimer levels may be helpful in supporting the diagnosis. ${ }^{7,8}$ A recent study found that elevated D-dimer levels may be a specific marker in the early recognition of acute SMV thrombosis, as well as predicting risk, outcomes, and treatment options. ${ }^{8}$ However, emergency physicians should maintain a high index of suspicion in patients with concerning features of the disease, since normal laboratory values, including lactate, do not reliably exclude the diagnosis.

Computed tomography scanning and CT angiography (CTA) are quite helpful in diagnosing MVT. Ultrasound of the upper abdomen may also play a role, noting dilated or thickened bowel wall with intraluminal air or echogenic material in the superior mesenteric vein or portal vein. ${ }^{9}$ Although magnetic resonance venography most reliably demonstrates thrombi, its lack of widespread availability makes CT with IV contrast the preferred initial study. ${ }^{3}$ Computed tomography not only has high sensitivity, but also offers alternative diagnoses in the undifferentiated presentation. ${ }^{1}$ One study found CT to be $100 \%$ sensitive in detecting any abnormality associated with MVT or bowel ischemia. ${ }^{10}$ Common CT findings of MVT include dilated and thickened bowel loops, mesenteric fat standing, ascites, a halo or target appearance of bowel, vessel filling defects from a thrombus, and pneumatosis intestinalis. ${ }^{11}$ The latter usually indicates transmural infarction, and can extend as portomesenteric vein gas. ${ }^{11}$ Of note, if the initial CT scan is non-diagnostic and a high clinical suspicion for mesenteric ischemia remains with no alternative diagnosis, CTA is the gold standard. ${ }^{3,7}$ Expeditious diagnosis of MVT is imperative, given the potential complications of intestinal infarction, submucosal hemorrhage secondary to edema, and third spacing of the venous outflow into the bowel wall due to collateral vessels being unable to redirect blood flow in conjunction with complete venous occlusion. ${ }^{12}$

Not all MVTs progress to infarction, given the extensive collateral circulation. Early diagnosis, however, is crucial for conservative management to be effective. ${ }^{9}$ Acute MVT without signs of infarction necessitates anticoagulation therapy to decrease clot propaga- 
tion and recurrence. ${ }^{1}$ In addition, prophylactic antibiotics to limit bacterial translocation, and bowel rest are advised. ${ }^{13,14}$ If the patient is unresponsive to anticoagulation, thrombolytic and endovascular therapies may be of benefit in select patients. ${ }^{15}$ Once intestinal ischemia or infarction develops, the prognosis is poor: mortality approaches $75 \%$ with infarction. ${ }^{1}$ If signs of bowel infarction are present, a laparotomy must be performed promptly, although in most cases, delayed patient presentation makes small bowel resection unavoidable. ${ }^{9}$ Further testing for hypercoagulability is recommended, particularly in isolated thrombosis, since long-term anticoagulation therapy may be necessary if a coagulopathy is discovered. ${ }^{1}$

\section{Conclusion}

Mesenteric venous thrombosis is atypical in a young, healthy patient. However, due to high mortality rates with disease progression, it is important to consider in any patient with unrelenting abdominal pain and vague gastrointestinal symptoms of uncertain cause, even in those without risk factors. Early detection and management of MVT before progression to mesenteric ischemia and infarction considerably lowers the mortality rate. Emergency physicians must be vigilant when treating a patient with abdominal pain out of proportion to physical examination, unrelenting pain despite analgesic medications, or repeat ED visits for the same abdominal complaints.

\section{References}

1. Harnik IG, Brandt LJ. Mesenteric venous thrombosis. Vasc Med. 2010;15(5):407-418. doi:10.1177/1358863x10379673.

2. Tilsed JV, Casamassima A, Kurihara H, et al. ESTES guidelines: acute mesenteric ischaemia. Eur J Trauma Emerg Surg. 2016;42(2):253-270. doi:10.1007/s00068-016-0634-0.

3. Tendler DA, Lamont JT, Grubel P. Mesenteric venous thrombosis in adults. UpToDate Web site. https://www. uptodate.com/contents/mesenteric-venous-thrombosis-in-adults. Accessed November 16, 2017.

4. Al-Zahrani HA, Lindsay T. Mesenteric ischemia. In: Hall JB, Schmidt GA, Kress JP, eds. Principles of Critical Care. 4th ed. New York, NY: McGraw Hill; 2015:1036-1044.

5. Kumar S, Sarr MG, Kamath PS. Mesenteric venous thrombosis. N Engl J Med. 2001;345(23):1683-1688. doi:10.1056/nejmra010076.

6. Al-Khazraji A, Hasan AQ, Patel I, Alkhawam H, Ghrair F, Lieber J. The role of abdominal computed tomography scan in acute pancreatitis. Pancreas. 2017;46(6):e52-e54. doi:10.1097/mpa.0000000000000837.

7. Bradbury MS, Kavanagh PV, Bechtold RE, et al. Mesenteric venous thrombosis: diagnosis and noninvasive imaging. Radiographics. 2002;22(3):527-541.

8. Yang S, Fan X, Ding W, et al. D-dimer as an early marker of severity in patients with acute superior mesenteric venous thrombosis. Medicine (Baltimore). 2014;93(29):e270. doi:10.1097/md.0000000000000270.

9. Matos C, Van Gansbeke D, Zalcman M, et al. Mesenteric vein thrombosis: early CT and US diagnosis and conservative management. Gastrointest Radiol. 1986;11(4):322-325.

10. Rhee RY, Gloviczki P, Mendonca CT, et al. Mesenteric venous thrombosis: still a lethal disease in the 1990s. J VasC Surg. 1994;20(5):688-697.

11. Furukawa A, Kanasaki S, Kono N, et al. CT diagnosis of acute mesenteric ischemia from various causes. AJR Am J Roentgenol. 2009;192(2):408-416. doi:10.2214/ajr.08.1138.

12. Johnson CC, Baggenstoss AH. Mesenteric vascular occlusion; study of 99 cases of occlusion of veins. Proc Staff Meet Mayo Clin. 1949;24(25):628-636.

13. Hmoud B, Singal AK, Kamath PS. Mesenteric venous thrombosis. J Clin Exp Hepatol. 2014;4(3):257-263. doi:10.1016/j.jceh.2014.03.052.

14. Schoots IG, Koffeman GI, Legemate DA, Levi M, van Gulik TM. Systematic review of survival after acute mesenteric ischaemia according to disease aetiology. Br J Surg. 2004;91(1):17-27.

15. Yang S, Fan X, Ding W, et al. Multidisciplinary stepwise management strategy for acute superior mesenteric venous thrombosis: an intestinal stroke center experience. Thromb Res. 2015;135(1):36-45. doi:10.1016/j. thromres.2014.10.018. 